\title{
Prospecção Tecnológica sobre Sistemas de Valoração de Tecnologias Protegidas por Patentes e/ou Registros de Programa de Computador
}

\author{
Technological Prospection on Technology Valuation System Protected by \\ Patents and/or Computer Program Records
}

\author{
Antonio Fredson de Sá Novaes ${ }^{1}$ \\ Gilton José Ferreira da Silva ${ }^{2}$ \\ Vivianni Marques Leite dos Santos ${ }^{1}$ \\ ${ }^{1}$ Universidade Federal do Vale do São Francisco, Petrolina, PE, Brasil \\ ${ }^{2}$ Universidade Federal de Sergipe, São Cristóvão, SE, Brasil
}

\begin{abstract}
Resumo
A partir das dificuldades identificadas nos processos de valoração de tecnologias, incluindo demanda interna do NIT/ Univasf, esta pesquisa tem como objetivo prospectar sistemas de valoração de tecnologias de forma a atender a essa necessidade. Para tanto, foram realizadas revisão de literatura científica sobre o tema, busca de patentes nacionais e internacionais, além da procura de softwares registrados no Brasil e em bases públicas. Não foram identificadas patentes e nem Registros de Programa de Computador (RPC) que permitam a valoração de novas tecnologias, cujo processo inclua etapas de apoio aos usuários e permita interação do gestor de inovação com os inventores. A partir da matriz SWOT e de Roadmap tecnológico, verificou-se ambiente favorável ao desenvolvimento e oportunidade de mercado para um sistema que implemente um processo de valoração amigável para usuários de várias áreas de formação.
\end{abstract}

Palavras-chave: Sistema de Valoração. Patentes. Registros de Programa de Computador.

\begin{abstract}
Based on the existing difficulties in the technology valuation processes, including internal demand from NIT/UNIVASF, this research aims to prospect systems of valuation of technologies to solve this necessity. To this, scientific literature review was carried out, as well as the search for national and international patents, in addition to the search for software registered in Brazil and on public bases. No patents were identified, nor were Computer Program Registries (RPC), which allow the valuation of new technologies, the process of which includes steps to help users and allow interaction between the innovation manager and the inventors. From the technological SWOT and ROADMAP matrix, there is a favorable environment for development and market opportunity for a system that implements a friendly valuation process for users from various graduation.
\end{abstract}

Keywords: Valuation System. Patents. Computer Program Registry.

Área Tecnológica: Prospecção Tecnológica. Valoração de Tecnologia. Tecnologia da Informação. 


\section{Introdução}

O desenvolvimento da Propriedade Intelectual (PI), principalmente nas instituições de ensino superior, pode contribuir sobremaneira para geração do conhecimento no sentido de evoluir nas mais diversas técnicas e na proposição de novas tecnologias de tal modo que tem grande potencial para melhoria da qualidade de vida da população. Entretanto, para que uma tecnologia chegue ao setor produtivo, faz-se necessário que sejam definidos os custos envolvidos na sua produção, o que pode constituir etapa limitante para que as referidas tecnologias sejam de fato utilizadas pela sociedade.

Buainain (2018) sustenta que a importância da PI transcende as polêmicas de que esta de fato constitua inovação, ou seja, chegue ao mercado, estando o Brasil ainda longe de acompanhar a produção mundial. Adiciona, ainda, que os mecanismos de proteção precisam ser aperfeiçoados, uma vez que se referem à propriedade dos ativos mais importantes para o funcionamento da economia contemporânea, observando também a liderança das instituições acadêmicas nos pedidos e nos registros de patentes dentro da baixa participação dos residentes em comparação à proteção de ativos intangíveis por empresas estrangeiras no país.

Como registrado por Cervantes e Lucarelli (2018), nota-se que há um consenso entre autores de renome de que a inovação é provocadora de desenvolvimento e de crescimento econômico, com alterações na sociedade e na vida das pessoas, sendo observadas como consequência das inovações. Devido à tal importância, Costa e Silva (2019) destacaram a proteção da PI como fator determinante para o desenvolvimento econômico, incentivando a criação de novos produtos ao garantir a devida remuneração ao criador, sendo também de grande importância a proteção dos direitos dos criadores de programa de computador, tendo em vista quanto a sociedade depende dessa tecnologia.

Por outro lado, Cruz et al. (2020) abordaram a complexidade envolvida nos processos de Transferência de Tecnologia (TT), os quais tornam a tecnologia acessível ao mercado. Eles informam ainda que, apesar de existir o incentivo à TT a partir da Lei de Inovação Federal (Lei n. 10.973/2004) e suas alterações, os Núcleos de Inovações Tecnológicos (NIT) estudados possuem pouca ou nenhuma informação quanto à transferência de tecnologia e de como seriam desencadeados esses processos (CRUZ et al., 2020).

Cavalcante, Almeida e Renault (2019), em pesquisa realizada na Fundação Oswaldo Cruz (Fiocruz), identificaram a cultura não orientada para TT, a morosidade do processo e a ausência de habilidades de valoração e de negociação como as principais barreiras dos processos de transferência tecnológica, podendo inclusive levar a instituição a realizar contratos que a prejudiquem. Outro estudo realizado na Universidade de Brasília (UnB) por Ferreira, Ghesti e Braga (2017) apresenta a falta de metodologia de valoração de tecnologias como fraqueza da instituição sobre os processos de TT, informando que o NIT carece do desenvolvimento de uma metodologia de valoração, assim como em muitos outros NITs do Brasil, relacionando diversos estudos que mostraram problema semelhante em outras universidades.

Leite et al. (2018) observaram que a valoração é um mecanismo importante para facilitar o processo de negociação, fornecendo referências de valores máximos e mínimos que auxiliam na precificação, com o objetivo de identificar o valor considerado justo, sendo útil tanto na comercialização e no licenciamento de tecnologia quanto na análise de risco e priorização em Pesquisa e Desenvolvimento (P\&D). Os autores verificaram ainda que a valoração pode ser 
medida usando múltiplas dimensões e que é consenso entre os estudiosos do tema que não existe um método único que deva ser utilizado para avaliar todas as tecnologias, confirmando os resultados por métodos alternativos, e que falhas na valoração podem resultar num parecer equivocado quanto ao real potencial tecnológico e mercadológico, comprometendo a negociação no futuro (LEITE et al., 2018).

Amaral et al. (2014) realizaram uma simulação que comparou a valoração de uma tecnologia protegida por patente com a valoração dessa mesma tecnologia considerando-a sem a proteção por patente, com os resultados indicando que, apesar de os custos de desenvolvimento serem maiores com a patente, os fluxos de caixa líquidos de tecnologia protegida por patente são bem superiores, obtendo um melhor saldo positivo e apontando, dessa forma, a importância da patente.

Ferreira e Souza (2019) também destacaram que a valoração é uma etapa fundamental para a negociação do invento, atribuindo um valor justo que represente a melhor descrição do potencial econômico de uma tecnologia, podendo ocorrer tanto para subsidiar a análise de viabilidade para decisão de investimento no desenvolvimento da tecnologia quanto no momento da negociação de transferência tecnológico.

Computadores, sistemas e internet, com o avanço da tecnologia da informação, são essenciais para muitas das tarefas realizadas no dia a dia, sendo o uso do software importante para a automatização das tarefas, melhorando a eficiência da empresa e a eficácia das atividades, com fácil utilização e lidando com muitas transações, o que pode ser visto como a simplificação de um processo (SARATKAR, 2019).

A maioria das empresas, seja de grande, médio ou pequeno porte, pública ou privada, já aderiu à informatização dos processos, precisando cada vez mais de softwares com qualidade, que atendam às suas necessidades gerenciais e operacionais, sendo esse um dos maiores problemas encontrados no mercado de software, ou seja, encontrar produtos de qualidade que atendam aos requisitos da empresa, sendo necessária a avaliação do software antes do seu lançamento no mercado (SILVA; MENDES; VIANA, 2020).

A prospecção é um passo importante no planejamento, com o objetivo de criar, explorar e testar os futuros possíveis e desejáveis para melhorar as decisões, desse modo, existem vários critérios possíveis para selecionar as técnicas, não sendo possível considerar uma família como melhor, uma vez que todas apresentam vantagens e desvantagens, assim é possível deduzir que a utilização de técnicas combinadas é uma medida vantajosa em estudos de prospecção (REIS; VINCENZI; PUPO, 2016). Observa-se ainda que os avanços tecnológicos na área de informática têm feito com que os autores de programas de computador busquem mecanismos de proteção que possam assegurar e garantir os direitos sobre suas criações (NETO et al., 2016).

Com a finalidade de identificar os desenvolvimentos tecnológicos relacionados à valoração de tecnologias, foi realizado um estudo prospectivo com o propósito de auxiliar futuras pesquisas, desenvolvimentos e inovações, tanto na academia quanto no mercado, incluindo análise SWOT final, com discussão sobre as forças e fraquezas, bem como ameaças e oportunidades para esse tipo de desenvolvimento, e Roadmap tecnológico com planejamento a curto, médio e longo prazos, considerados os produtos a serem desenvolvidos, as tecnologias necessárias e os mercados consumidores e concorrentes, para o desenvolvimento de um sistema computacional que confira maior celeridade a um processo de valoração com pedido de patente a partir de um dos autores deste artigo. 


\section{Metodologia}

O estudo tem natureza aplicada, gerando conhecimentos quanto à valoração de tecnologias para aplicação em curto prazo, visto que fornece resultados para desenvolvimento de tecnologia pelos autores. Tem abordagem quali-quantitativa e objetivo exploratório, com busca de informações na literatura, utilizando técnicas de pesquisa documental e bibliográfica.

A pesquisa bibliográfica na literatura científica foi realizada nas bases do Periódico CAPES, Google Acadêmico e LENS.ORG, em março de 2021, sendo utilizada inicialmente uma expressão mais genérica (1) e, em seguida, uma expressão com palavras combinadas (2), com busca em quaisquer campos, tal como previsto na busca avançada padrão.

A pesquisa de patentes foi realizada no software de busca e de tratamento de patentes obtidas em metabases, o Questel Orbit, em março de 2021, utilizando a Expressão (1) e a Expressão (3) no título e resumo, sendo também realizada a busca na base nacional a partir do site do Instituto Nacional da Propriedade Industrial (INPI) com a expressão "valoração" no título e no resumo. Observa-se que as Expressões (2) e (3) são semelhantes, apenas com troca das aspas duplas (") por aspas simples ("). Foi considerada a busca por família de patentes, uma vez que por patentes individuais seria obtido um maior quantitativo, ao passo que uma patente pode ser protegida em um conjunto de países, constituindo, dessa forma, uma família.

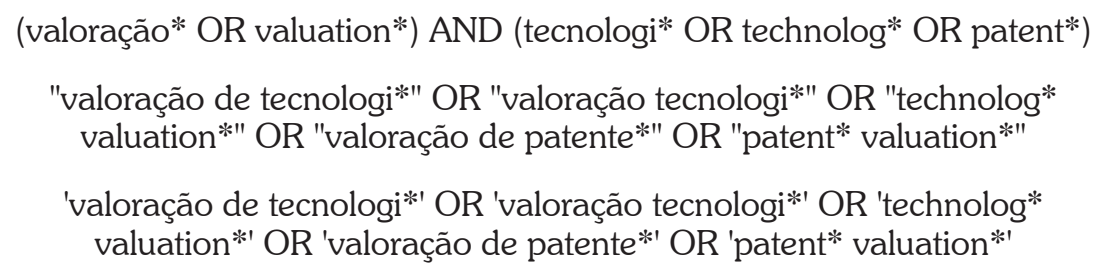

Foi realizada também uma busca na base de dados do INPI referente aos registros de programas de computador com o objetivo de valorar tecnologias em março de 2021, utilizando a palavra-chave "valor*" no título, e ainda os termos valor, patente e tecnologia no portal do Software Público Brasileiro, sendo utilizado um termo de cada vez.

Em etapa pós-prospectiva, foi elaborada a matriz SWOT com os aspectos internos e externos que influenciam o desenvolvimento de uma nova tecnologia para a valoração tecnológica, seja patente ou programa de computador, com o objetivo de auxiliar o planejamento de desenvolvimento de um sistema com essa finalidade. Segundo Souza et al. (2021), a matriz SWOT pode contribuir para estabelecer os objetivos estratégicos e colaborar para que a tecnologia possa chegar à sociedade, elencando as forças, as oportunidades, as fraquezas e as ameaças.

O desenvolvimento do Roadmap tecnológico, em conformidade com a matriz SWOT, complementa a pós-prospecção, permitindo a visualização das tecnologias, dos produtos/processos e do mercado em curto, médio e longo prazos, o que possibilita, segundo Barro, Santos e Azevedo (2020), um planejamento estratégico dos principais interesses relacionados à pesquisa $e$, ainda, apresenta o estado da arte. 


\section{Resultados e Discussão}

Em pesquisa bibliográfica na literatura utilizando a Expressão (1), foram encontrados 120.276, 16.600 e 25.869 registros nas bases dos Periódicos CAPES, Google Acadêmico e LENS. ORG (em 28/03/2021), respectivamente (Tabela 1). Porém observou-se que vários resultados não estavam realmente relacionados à valoração de tecnologia por meio de verificação do título e do resumo. Repetindo a pesquisa bibliográfica, utilizando a Expressão (2), foram encontrados 689, 290 e 177 registros nas bases do Periódicos CAPES, Google Acadêmico e LENS.ORG, respectivamente (Tabela 1), o que melhorou o resultado por meio da busca com termos diretos.

Tabela 1 - Quantidade de registros em pesquisa bibliográfica por fonte e expressão utilizada

\begin{tabular}{ccc} 
Fonte & Quantitativo Pela Expressão (1) & Quantitativo PEla Expressão (2) \\
Periódicos CAPES & 120.276 & 689 \\
Google Acadêmico & 16.600 & 290 \\
LENS.ORG & 25.869 & 177 \\
\hline
\end{tabular}

Fonte: Elaborada pelos autores deste artigo a partir de dados da base de Periódicos CAPES, Google Acadêmico e LENS.ORG (2021)

Considerando estes últimos resultados do Periódicos CAPES, a maioria dos registros encontrados está em inglês (609) e apenas 11 estão em português (1,6\%), com publicação entre 1972 e 2021. Destaca-se que 129 registros são sobre Valoração (Valuation); 33 sobre Transferência de Tecnologia (Technology Transfer); 29 sobre Valoração de Patente (Patent Valuation); e 28 sobre Valoração de Tecnologia (Technology Valuation) (Gráfico 1), observa-se que um registro pode estar associado a mais de um tópico.

Gráfico 1 - Quantidade de registros por tópico no Periódico CAPES

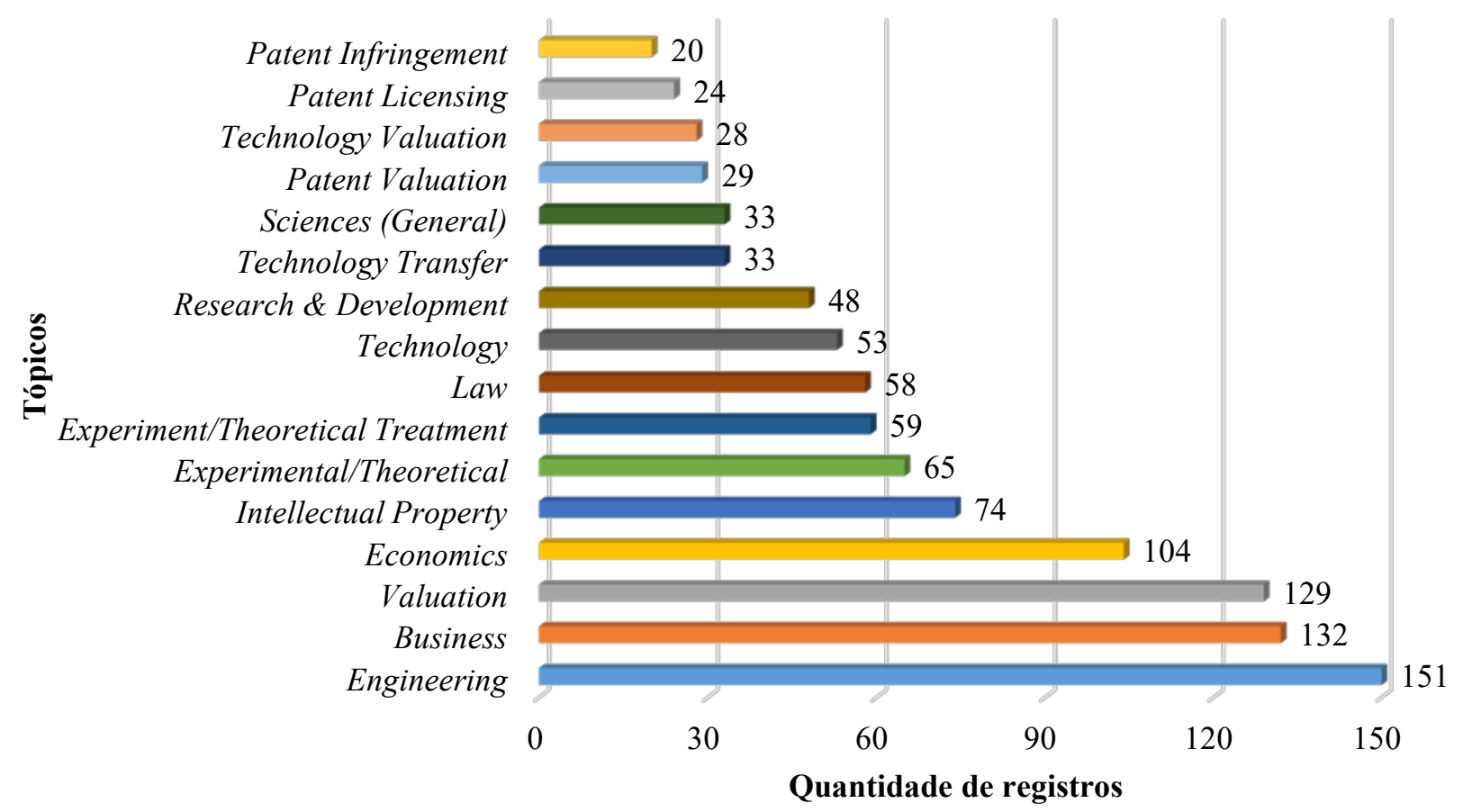

Fonte: Elaborado pelos autores deste artigo a partir da base de Periódicos CAPES (2021) 
Ainda sobre a quantidade de registros encontrados por tópico (Gráfico 1), observa-se que as maiores quantidades de registros estão relacionadas à engenharia (Engineering) com 151, seguido pelos registros relacionados a negócios (Business) com 132, estando a valoração (Valuation) em terceiro lugar (129), ao utilizar "valoração de tecnologi*" OR "valoração tecnologi*" OR "technolog* valuation*" OR "valoração de patente*" OR "patent* valuation*" como palavras-chave.

A análise sobre a evolução das publicações ao longo do tempo (Gráfico 2) permitiu constatar que a maioria dos registros na base de Periódicos da CAPES (628) foi publicada nos últimos 20 anos, observando que a quantidade de registros vem aumentando, porém com uma queda acentuada em 2020. Supõe-se que esse decréscimo tem como influência os efeitos a partir das medidas de isolamento exigidas devido à pandemia da COVID-19. Ademais, nos três primeiros meses do ano de 2021 (até 28 de março), foram publicados 12 registros, para o qual estima-se retomada do crescimento de publicações, o que pode estar relacionado à adaptação dos pesquisadores a esse novo normal.

Gráfico 2 - Quantidade de registros nos últimos 20 anos na base de Periódicos CAPES

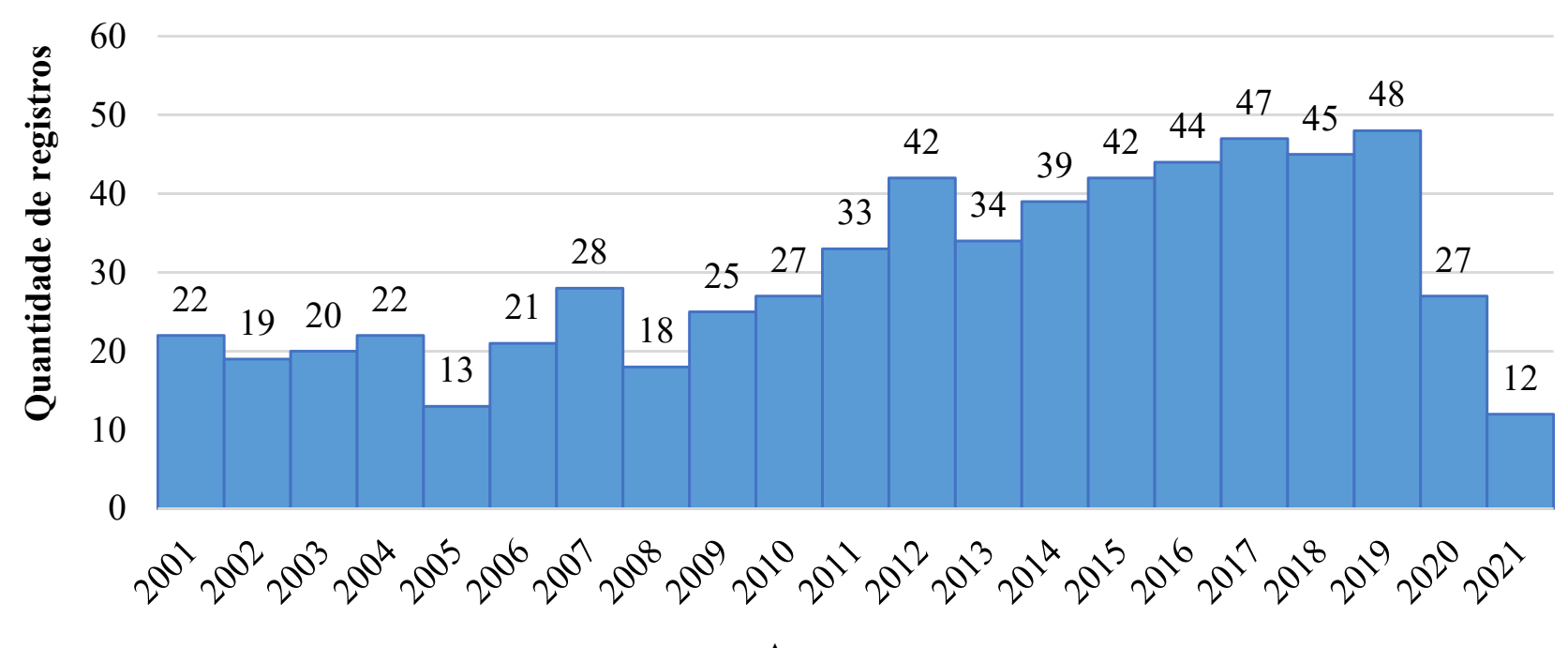

Anos

Fonte: Elaborado pelos autores deste artigo a partir da base de Periódicos CAPES (2021)

A pesquisa no Orbit (acesso em 29/03/2021) com a Expressão (1) no título e no resumo permitiu identificar 336 famílias de patentes, destas, 175 ainda ativas (103 concedidas e 72 pendentes) (Tabela 2) com a maioria dos registros relacionados a modelos e métodos de valoração de tecnologias e patentes. A busca com a Expressão (3) no título, no resumo ou na descrição encontrou 283 famílias de patentes, sendo 146 ativas (104 concedidas e 42 pendentes). Das 283, apenas oito foram publicadas no Brasil, porém nenhuma destas últimas realmente estava relacionada à valoração de tecnologias/patentes. 
Tabela 2 - Quantidade de famílias de patentes no Orbit por situação e pesquisa utilizada

\begin{tabular}{cccccc} 
Pesquisa & Total & InATIVA & Ativa & ConcedidA & Pendente \\
Expressão (1): título, resumo e descrição & 23.669 & 11.034 & 12.635 & 8.788 & 3.847 \\
Expressão (1): título e resumo & 336 & 161 & 175 & 103 & 72 \\
Expressão (3): título, resumo e descrição & 283 & 137 & 146 & 104 & 42 \\
$\begin{array}{c}\text { Expressão (3) AND software: } \\
\text { título, resumo e descrição }\end{array}$ & 37 & 18 & 19 & 13 & 6 \\
\hline
\end{tabular}

Fonte: Elaborada pelos autores deste artigo a partir de dados do Orbit (2021)

Ao incluir "AND software" na última busca, o resultado foi reduzido para 37 famílias de patentes, sendo 19 ativas e apenas 13 concedidas. A análise das 19 famílias de patentes (Quadro 1) pelo título e, em seguida, pela descrição aponta apenas três famílias de patente relacionadas a métodos e técnicas para softwares de valoração, constituindo: US20130073439, relacionada à técnica para avaliação de ativos intangíveis usando índices financeiros, que está pendente de análise na Índia, porém retirada da China e anulada nos Estados Unidos por constar assunto óbvio e ideias abstratas; EP1525540, relacionada ao uso de técnicas de valoração baseadas no modelo de Precificação de Opções de Blach-Sholes ou métodos de fluxo de caixa descontado, a qual se encontra concedida nos Estados Unidos, com previsão de expiração em 2024; e US20150199781, segundo a qual o preço do ativo de patente é determinado com base no lucro para o setor e na métrica de contribuição, que se encontra concedida no Japão e na Coreia, porém anulada nos Estados Unidos com justificativa de constituir assunto óbvio e outros aspectos não bem definidos e não inovadores.

Quadro 1 - Análise das famílias de patentes ativas no Orbit com Expressão (3) AND software

\begin{tabular}{|c|c|c|}
\hline $\begin{array}{l}\text { Data da } \\
\text { primeira } \\
\text { prioridade }\end{array}$ & Título & Análise da patente \\
\hline 05/12/2000 & $\begin{array}{l}\text { (US7653551) } \\
\text { Method and system for searching } \\
\text { and submitting online via } \\
\text { an aggregation portal }\end{array}$ & $\begin{array}{l}\text { Método e sistema para procura de propriedade } \\
\text { intelectual, bens e serviços oferecidos para venda } \\
\text { on-line. O método realiza a busca de acordo } \\
\text { com alguns critérios e apresenta a lista } \\
\text { de propriedades intelectuais com opção } \\
\text { de formatação e de ordenamento. }\end{array}$ \\
\hline 29/06/2001 & $\begin{array}{l}\text { (EP1525540) } \\
\text { Simultaneous intellectual property } \\
\text { search and valuation system } \\
\text { and methodology (sips-vsm) }\end{array}$ & $\begin{array}{l}\text { Sistema, método e fluxo lógico baseado em } \\
\text { computador, habilitada para web, que, entre } \\
\text { outras funções, realiza a busca de propriedade } \\
\text { intelectual e utiliza técnicas de valoração baseadas } \\
\text { no modelo de Precificação de Opços de Blach- } \\
\text { Sholes ou métodos de fluxo de caixa descontado. } \\
\text { Patente concedida apenas nos Estados Unidos } \\
\text { e com previsão de expiração em } 2024 \text {. }\end{array}$ \\
\hline 08/04/2005 & $\begin{array}{l}\text { (US8355932) } \\
\text { System and method for managing } \\
\text { intellectual property-based risks }\end{array}$ & $\begin{array}{l}\text { Métodos e sistemas para gerenciamento de riscos } \\
\text { de cobertura de patentes. Realiza busca de patentes } \\
\text { para verificar responsabilidade monetária por } \\
\text { violação de patente e determinar valor justo de } \\
\text { royalty ao proprietário da primeira patente. }\end{array}$ \\
\hline $22 / 06 / 2006$ & $\begin{array}{l}\text { (US20090276849) } \\
\text { Intellectual property managing } \\
\text { system, intellectual property managing } \\
\text { method, and program for the same }\end{array}$ & $\begin{array}{l}\text { Sistema de gerenciamento de propriedade } \\
\text { intelectual com compartilhamento de } \\
\text { informações entre empresas. }\end{array}$ \\
\hline
\end{tabular}




\begin{tabular}{|c|c|c|}
\hline $\begin{array}{l}\text { Data da } \\
\text { primeira } \\
\text { prioridade }\end{array}$ & Título & Análise da patente \\
\hline 08/09/2008 & $\begin{array}{l}\text { (EP2340515) } \\
\text { A computer implemented system and } \\
\text { method for providing a community } \\
\text { and collaboration platform around } \\
\text { knowledge transfer, expertise, } \\
\text { innovation, tangible assets, intangible } \\
\text { assets and information assets }\end{array}$ & $\begin{array}{l}\text { Sistema implementado em computador para } \\
\text { promover a colaboração entre usuários, relacionado } \\
\text { à inovaçãao, bens tangíveis e intangíveis, entre outros. }\end{array}$ \\
\hline $17 / 05 / 2010$ & $\begin{array}{l}\text { (US20130073439) } \\
\text { Methods and systems for patent } \\
\text { valuation using financial ratios }\end{array}$ & $\begin{array}{c}\text { Técnica para avaliação de ativos intangíveis usando } \\
\text { índices financeiros. Patente retirada na China, anulada } \\
\text { nos Estados Unidos e ainda pendente na Îndia. }\end{array}$ \\
\hline 03/10/2011 & $\begin{array}{l}\text { (US10803073) } \\
\text { Systems, methods and user interfaces } \\
\text { in a patent management system }\end{array}$ & $\begin{array}{c}\text { Sistema e método para verificar a especificação } \\
\text { de uma patente automaticamente } \\
\text { por meio das reivindicações. }\end{array}$ \\
\hline 03/10/2011 & $\begin{array}{l}\text { (US10860657) } \\
\text { Patent mapping }\end{array}$ & Sistema e método para mapeamento de patentes. \\
\hline 24/02/2012 & $\begin{array}{c}\text { (US10380707) } \\
\text { Patent life cycle management system }\end{array}$ & $\begin{array}{l}\text { Método e sistema para gerenciar o custo e a qualidade } \\
\text { dos pedidos de patentes, com controle do ciclo } \\
\text { de vida do processo internacional de patentes. }\end{array}$ \\
\hline 29/08/2012 & $\begin{array}{l}\text { (US9461876) } \\
\text { System and method for fuzzy concept } \\
\text { mapping, voting ontology crowd } \\
\text { sourcing, and technology prediction }\end{array}$ & $\begin{array}{l}\text { Sistema para capacitação e compartilhamento } \\
\text { de informações que, entre outros dados, pode } \\
\text { ser encontrado o valor previsto da tecnologia. }\end{array}$ \\
\hline $14 / 01 / 2014$ & $\begin{array}{l}\text { (US20150199781) } \\
\text { Patent Valuation System }\end{array}$ & $\begin{array}{l}\text { Métodos e sistemas para avaliação de ativos } \\
\text { de patentes. O preço para o ativo de patente é } \\
\text { determinado com base no lucro para o setor e na } \\
\text { métrica de contribuiçãa. Patente concedida no Japão } \\
\text { e na Coreia, porém anulada nos Estados Unidos. }\end{array}$ \\
\hline $31 / 03 / 2014$ & $\begin{array}{l}\text { (US20200358749) } \\
\text { System and method for providing } \\
\text { multiple application programming } \\
\text { interfaces for a browser to manage } \\
\text { payments from a payment service }\end{array}$ & Sistema e método para gestão de pagamentos. \\
\hline $31 / 03 / 2014$ & $\begin{array}{l}\text { (US10621653) } \\
\text { System and method for providing } \\
\text { payments for users in connection } \\
\text { with a device software module } \\
\text { having a payment application } \\
\text { programming interface }\end{array}$ & $\begin{array}{l}\text { Interface de programação de aplicativos } \\
\text { (API) para navegador web que permite ao } \\
\text { usuário escolher a forma de pagamento. }\end{array}$ \\
\hline $19 / 05 / 2014$ & $\begin{array}{c}\text { (US10095388) } \\
\text { Configurable patent strength calculator }\end{array}$ & $\begin{array}{l}\text { Sistema para determinar a pontuação da } \\
\text { força de uma patente que determina a } \\
\text { probabilidade do direito ser mantido. }\end{array}$ \\
\hline 04/05/2017 & $\begin{array}{l}\text { (EP3619627) } \\
\text { Providing cryptocurrency payments } \\
\text { through a browser application } \\
\text { programming interface }\end{array}$ & Sistema e método para recebimento de pagamentos. \\
\hline $23 / 10 / 2017$ & $\begin{array}{l}\text { (US20200327629) } \\
\text { System and Method of IP Ownership } \\
\text { and IP Registration Via a Blockchain } \\
\text { Transactional Platform }\end{array}$ & $\begin{array}{l}\text { Plataforma para contratos de Propriedade Intelectual, } \\
\text { com informações sobre a patente, incluindo o } \\
\text { valor, porém não é realizada a valoração. }\end{array}$ \\
\hline
\end{tabular}




\begin{tabular}{|c|c|c|}
\hline $\begin{array}{l}\text { Data da } \\
\text { primeira } \\
\text { prioridade }\end{array}$ & Título & Análise da patente \\
\hline 09/11/2017 & $\begin{array}{l}\text { (KR101836806) } \\
\text { Technology evaluation model generation } \\
\text { method reflecting characteristic of marine } \\
\text { and fishery field and device thereof }\end{array}$ & $\begin{array}{l}\text { Modelo de avaliação de tecnologia por } \\
\text { meio de pontuação de indicadores. }\end{array}$ \\
\hline 23/08/2018 & $\begin{array}{l}\text { (CN109102197) } \\
\text { Patent value evaluation system }\end{array}$ & $\begin{array}{l}\text { Estimativa de avaliação de valor por meio de busca } \\
\text { bibliográfica e com análise comparativa da patente. }\end{array}$ \\
\hline $11 / 04 / 2019$ & $\begin{array}{l}\text { (US20210089653) } \\
\text { System and method } \\
\text { employing virtual ledger }\end{array}$ & $\begin{array}{l}\text { Sistema com dispositivo para avaliação de ativos } \\
\text { com comunicação pela internet a partir das } \\
\text { informações dos ativos e com base em votação } \\
\text { sendo retornado um sinal de avaliação, não } \\
\text { sendo, portanto, para valoração de tecnologias. }\end{array}$ \\
\hline
\end{tabular}

Fonte: Elaborado pelos autores deste artigo a partir de dados do Orbit (2021)

As 18 famílias de patentes inativas, considerando a Expressão (3) AND software (Tabela 2), não estão relacionadas a software de valoração. Entre estas, apenas uma tem a situação de expirada, sendo relacionada a método e sistema para exibir informações de progresso de aplicativo e meio de armazenamento. As outras 17 estão com situação caducada e relacionadas à avaliação de imóveis, à avaliação de ideias para tecnologias, a métodos para marketing, ao licenciamento e trocas de patentes, à gestão de riscos de patentes e à negociação de propriedade intelectual.

Com base na análise do domínio tecnológico das famílias de patentes localizadas a partir do Orbit (Gráfico 3), considerando ativas e inativas, conclui-se que a maioria envolve Métodos de TI para Gestão (IT Methods for Management), com 228 famílias, e tecnologias de computador (Computer Technology) em segunda lugar, com 56 famílias, ao considerar 'valoração de tecnologi*' OR 'valoração tecnologi*' OR 'technolog* valuation*' OR 'valoração de patente*' OR 'patent* valuation*' como palavras-chave. Ao adicionar "AND software" à expressão, o resultado é reduzido para 35 e nove famílias, respectivamente. Observa-se que muitas dessas patentes são relacionadas a métodos que podem ser aplicados em programas de computador.

Gráfico 3 - Quantidade de famílias de patentes por domínio tecnológico

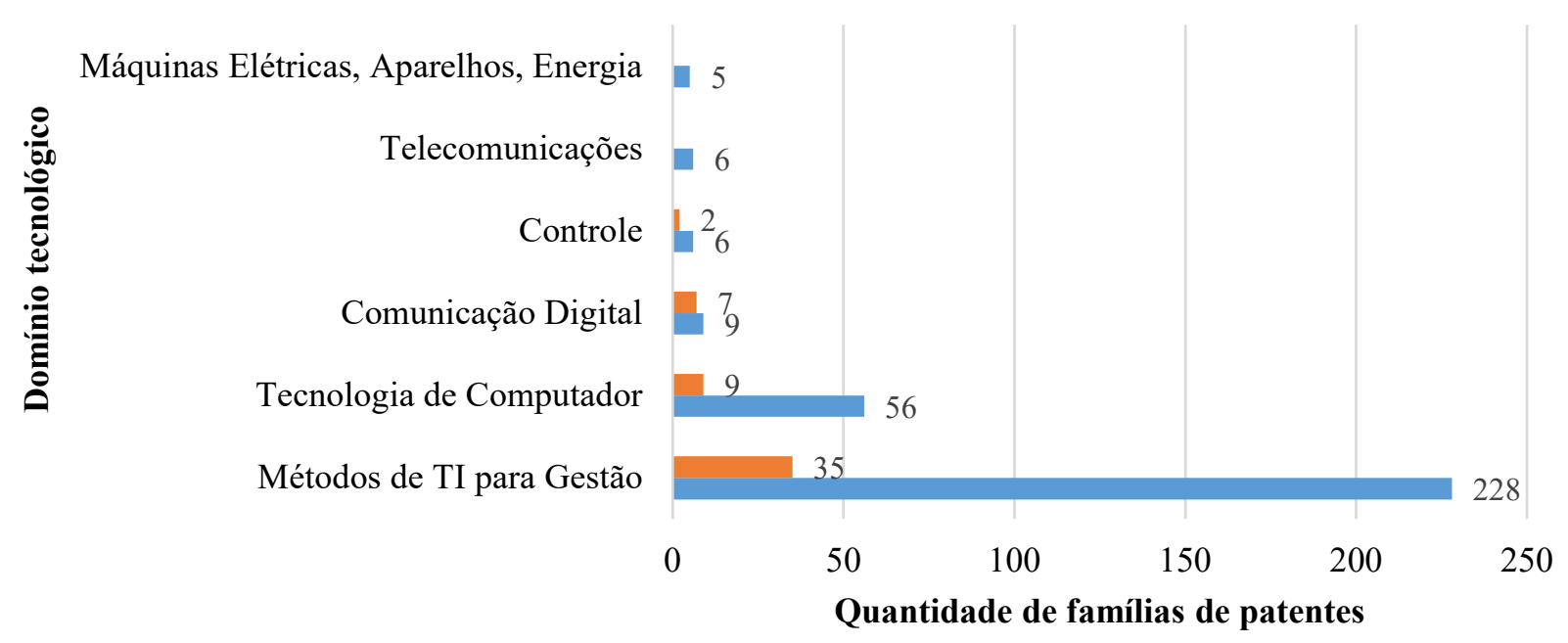

$$
\text { Expressão (3) AND software } \quad \text { Expressão (3) }
$$

Fonte: Elaborado pelos autores deste artigo a partir de dados do Orbit (2021) 
Quanto ao país de primeira prioridade (Gráfico 4), considerando ativas e inativas, pode-se observar um empate técnico entre a Coreia e os Estados Unidos, com aproximadamente 100 famílias de patentes, ao considerar a Expressão (3). Porém, ao adicionar "AND software" a essa expressão, os Estados Unidos ficam com a maior quantidade de famílias de patentes (30), e a Coreia com apenas uma. Acrescenta-se à discussão a observação de que, entre as 283 famílias de patentes contendo a Expressão (3), há oito famílias com pedido de proteção no Brasil, porém nenhuma delas configura primeira prioridade no Brasil, destacando-se, ainda, que são encontrados apenas 10 países de primeira prioridade para a Expressão (3) e apenas seis ao adicionar "AND software" à expressão.

Gráfico 4 - Quantidade de famílias de patentes por país de primeira prioridade

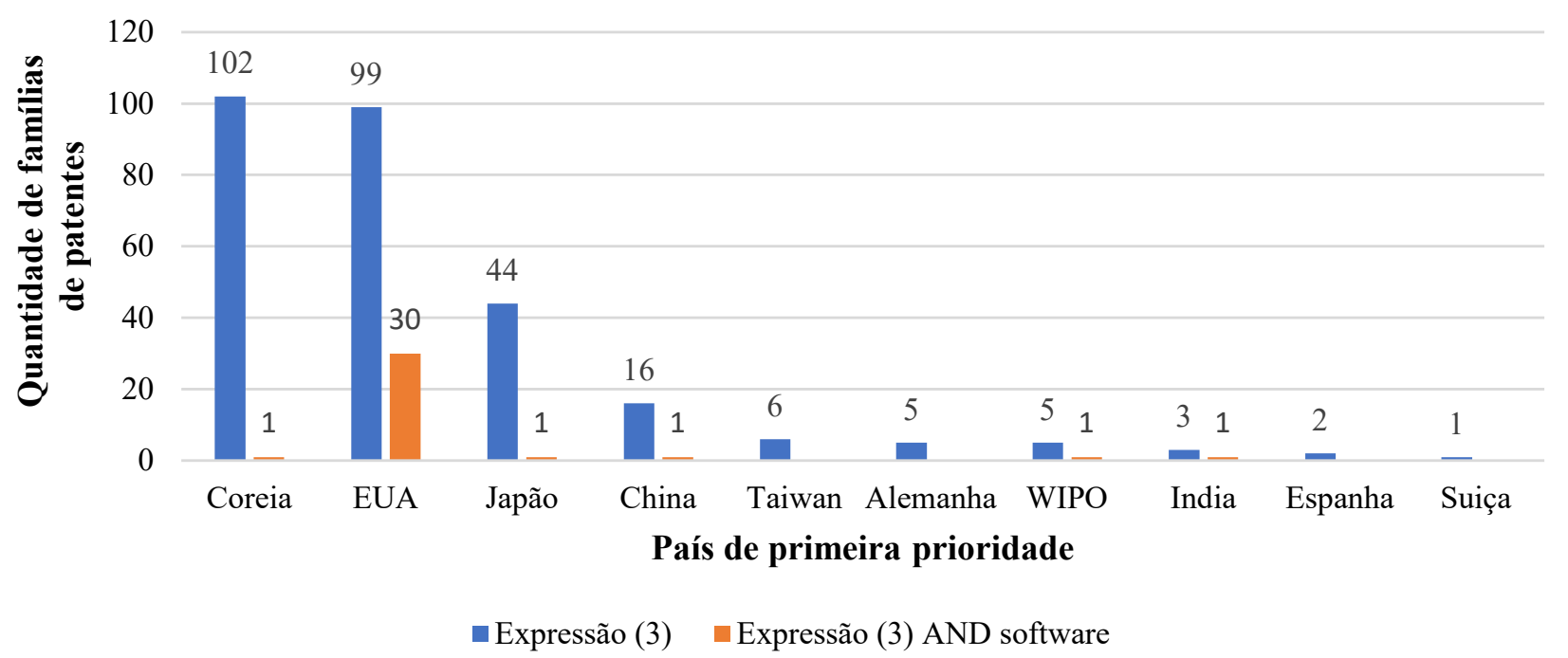

Fonte: Elaborado pelos autores deste artigo a partir de dados do Orbit (2021)

A procura por patentes na base do Instituto Nacional da Propriedade Industrial (INPI), com o termo "valoração", permitiu identificar apenas quatro registros de pedidos de patentes pelo título e 20 pelo resumo (incluídos os quatro registros verificados na busca pelo título), porém, após análise detalhada do título e na descrição dessas patentes, verificou-se que nenhuma delas está relacionada à valoração de tecnologias protegidas por patentes, por exemplo, destacando aquelas com termo "valor*" no título: patentes para avaliação valoração de bens rurais; valoração de efluentes do setor sucroenergético; método para determinação do índice valor ambiental; processo para valoração petrofísica de formação de subsolo; e valoração dinâmica de anúncios em sistema de transmissão de dados.

A busca de programas de computador com certificado de registro no INPI, contendo a palavra-chave "valor*" no título do programa, trouxe 41 resultados, porém a maioria não está relacionada à valoração de tecnologias quando analisados, detalhadamente, o título e o campo de aplicação. Alguns exemplos de programas são relativos à calculadora de Imposto de Renda (IR), apuração de resultados em bolsas de valores, análise de fluxo de ativos em bolsa de valores, tratamento e aferição de valores ausentes, valorização de belezas cênicas, valor de bens rurais, auditoria de valor fiscal, apuração de valor adicionado fiscal, análise georreferenciada de valores de referência para a venda, valorização de carreiras, valorização de profissionais, valoração de investimentos, análise de valor financeiro, buscador de voo, valores mobiliários, 
valorização do bem e, ainda, programas para administração de patrimônio, contabilidade, marketing, automação comercial e gerenciador de informações.

Houve maior dificuldade para obter mais detalhes sobre um programa de computador com título "valor app", o que foi possível somente ao realizar busca no Google com essa palavra-chave e, juntamente, a palavra "Tocantins", uma vez que se trata de programa de computador que tem como titular a Fundação Universidade Federal do Tocantins, verificando-se que se trata de "Gerenciamento e Administração de Precificação de Obras Artísticas e Artesanais".

Em suma, com relação aos programas de computador registrados no INPI, notou-se apenas um registro considerado com o título "Valoração Tecnológica Qualitativa - Fase 1", da Universidade Federal de Santa Maria (UFSM), com depósito em 05/12/2018 e registro em 11/12/2018 (BR 512018052286 6). Ademais, não foram encontradas informações adicionais sobre esse programa de computador quando pesquisada a expressão "Valoração Tecnológica Qualitativa" no Google, a não ser uma página da UFSM referente a um dos inventores que classifica o software como "Outros tipos" ao invés de "Computacional" (UFSM, 2021).

Acrescenta-se que, ao buscar o Currículo Lattes do professor/autor informado (LATTES, 2021), verificou-se que a modelagem e o sistema são projetos que ainda estão em andamento, informando um programa de computador para valorar tecnologias em empresas de base tecnológica e agências de transferência de tecnologias, "para mensurar monetariamente uma tecnologia a ser comercializada em um processo de transferência de tecnologia através da descrição das principais características dos processos de valoração existentes, identificação dos fatores mais relevantes para a valoração de tecnologias, modelagem com base nos fatores identificados e realização da programação da modelagem proposta pelo Núcleo de Inovação e Competitividade (NIC-UFSM)".

Na fase final da etapa prospectiva, a busca no portal do Software Público Brasileiro (BRASIL, 2021) permitiu identificar que não há nenhum registro ao utilizar qualquer um dos termos seguintes como filtro: valor, patente ou tecnologia. Observando que a pesquisa é realizada com o termo de forma parcial, ou seja, encontraria, por exemplo, as palavras valorar ou valoração por conterem "valor" como parte da palavra.

Em seguida, na etapa pós-prospectiva, passou-se para a identificação dos interferentes internos e externos ao desenvolvimento de uma nova tecnologia para valoração de patentes, elaborando-se a matriz SWOT (Figura 1). Entre os aspectos positivos do ambiente interno (as forças), destaca-se o conhecimento prévio das respectivas áreas pelos profissionais alocados para o desenvolvimento do Sistema, tal como especialista em desenvolvimento de softwares, especialistas em Propriedade Intelectual (PI) e também em valoração de patentes. 
Figura 1 - Matriz SWOT para desenvolvimento de sistema de valoração

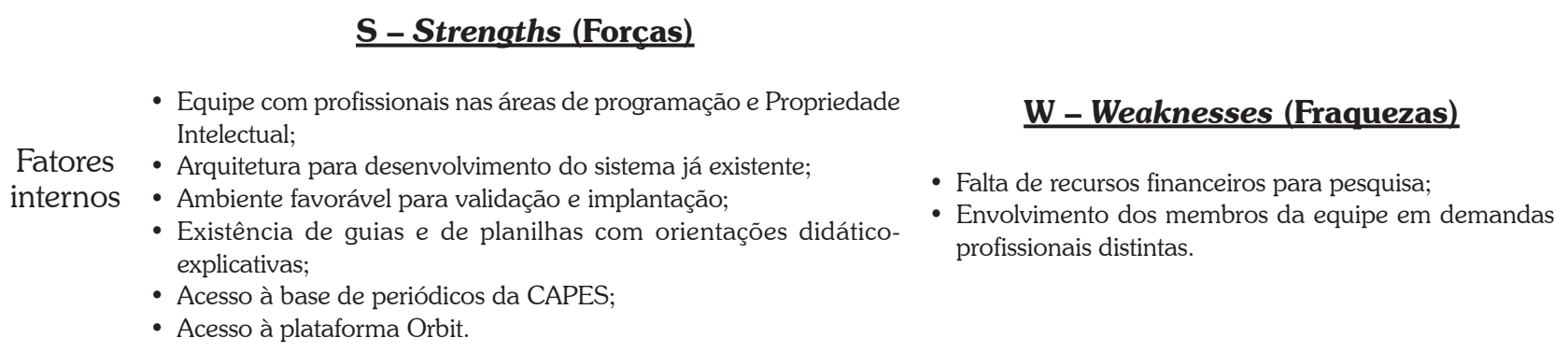

\section{O-Oportunities (Oportunidades)}

Fatores externos
Dificuldade existente na escolha e na utilização de metodologias de valoração;

- Produto inovador por falta de tecnologias que facilitam o processo de valoração de tecnologias.

\section{T-Threats (Ameacas)}

- Possível desenvolvimento da ferramenta por outra instituição;

- Uso ilegal da tecnologia, com pirataria ou cópia de informações.

Fonte: Elaborada pelos autores deste artigo (2021)

Do ponto de vista de infraestrutura física e de conteúdo técnico (Figura 1 - Forças), já há detalhamento quanto ao processo de valoração a ser implementado, para o qual foi solicitada proteção por patente (SANTOS et al., 2021); Guia Prático para Valoração de Patentes (BRITO; SILVA; SANTOS, 2021); planilha eletrônica, contendo as fórmulas necessárias para implementação dos métodos de abordagem de mercado e opções reais; bem como artigo com detalhamento acerca de aplicação do método APF (HÜLLER et al., 2021). Ademais, é importante mencionar que todas as fontes de dados para desenvolvimento do sistema contêm autoria de um dos responsáveis por este artigo, que orienta o projeto de mestrado do principal responsável pela tecnologia a ser desenvolvida.

Acrescenta-se ainda o interesse do NIT da Universidade Federal do Vale do São Francisco (Univasf) pelo sistema, de modo que foi escolhido para validação, e o gestor disponibilizou anuência tanto para a validação quanto para a implementação do sistema. A despeito disso, deve-se buscar minimizar os efeitos das "fraquezas" (ambiente interno) no cumprimento dos prazos do planejamento (ver Figura 2), quais sejam: a falta de recursos financeiros para a pesquisa e o envolvimento do desenvolvedor do sistema e dos demais membros da equipe em outras atividades profissionais, o que poderia ser resolvido com bolsas e empregos de terceiros no projeto. 
Figura 2 - Roadmap para desenvolvimento de sistema de valoração

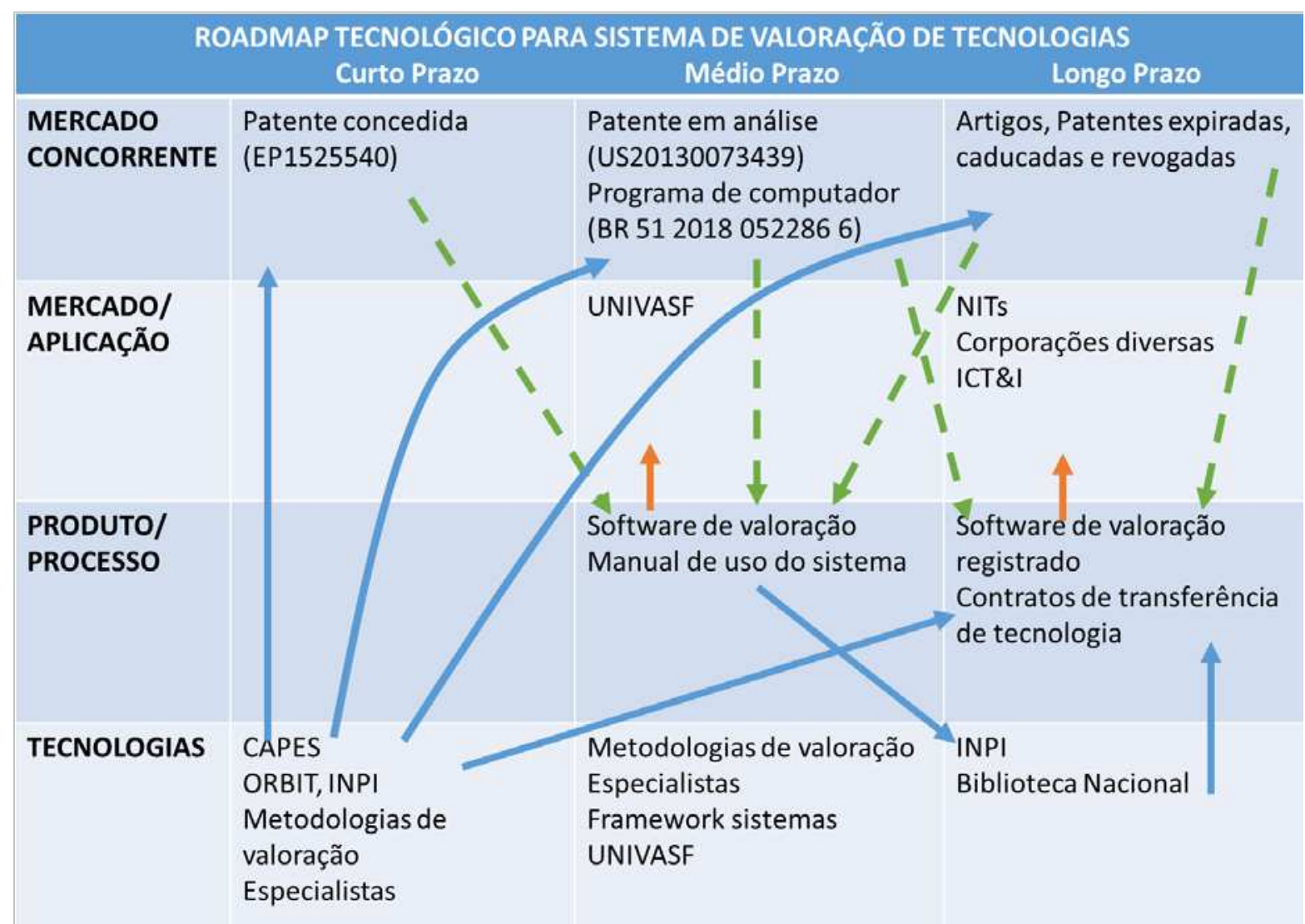

Fonte: Elaborada pelos autores deste artigo (2021)

Para o ambiente externo, existe a oportunidade associada à problemática sobre a escolha da metodologia e sua utilização de forma manual, que poderá ser suprida pelo novo software. Em contrapartida, observa-se como ameaça a possibilidade de outros sistemas serem desenvolvidos com a mesma finalidade de valoração, bem como o risco de cópias não autorizadas de toda a tecnologia ou de alguns processos que poderão reduzir possibilidades de negociações de transferência para outras instituições.

De posse dos resultados das buscas e matriz SWOT (Figura 1), foi elaborado o Roadmap tecnológico (Figura 2) a partir do qual se tem visão bastante rica e rápida acerca das tecnologias necessárias, produtos/processos a serem desenvolvidos, além dos mercados consumidores e concorrentes ao longo do desenvolvimento do sistema, nos horizontes de curto, médio e longo prazos (Figura 2) ao longo de um ano.

A partir da busca de patentes, no mercado concorrente, estão destacadas as patentes que, apesar de distintas, têm finalidades complementares ou relacionadas, mesmo que indiretamente, àquela específica deste desenvolvimento. Nesse ínterim, destaca-se que a nova proposta tem como principais elementos de novidade a funcionalidade de poder ser utilizado por qualquer pessoa, mesmo que não seja da área de ciências contábeis ou afins, devido à disponibilidade de notas didático-explicativas e de exemplos, com etapas que favorecem a participação do gestor/ administrador, mas também do autor/inventor, que tem posse de informações tecnológicas de uma tecnologia a ser valorada, com detalhes sobre o desenvolvimento (SANTOS et al., 2021).

A previsão da validação está a médio prazo (Figura 2), incluindo sua implementação no NIT da Univasf, sendo confeccionado um manual de utilização. Após o registro do software de 
valoração e da obtenção do ISBN para o manual, o novo processo e o sistema irão compor vitrine tecnológica da Univasf, com possibilidade de negociações formalizadas por meio de contratos de transferência de tecnologia com outros NITs, corporações diversas e Instituições de Ciência, Tecnologia e Inovação (ICT\&I), visto que a patente já se encontra em fase de análise no INPI, a qual foi citada no parágrafo anterior.

Acrescenta-se que a expectativa é de que o sistema seja validado e implementado durante uma disciplina do Mestrado Profissional em Propriedade Intelectual e Transferência de Tecnologia para a Inovação (Profnit/Univasf), que visa à execução de projeto para inovação em ambiente produtivo, nomeada oficina profissional. Assim, ao final da disciplina, o sistema estará disponível no NIT/Univasf, propiciando a obtenção de valores de referência para tecnologias protegidas por patente ou por registro de programa de computador, de forma amigável para os mais diversos gestores da inovação que liderem o setor, independentemente de suas formações acadêmicas.

\section{Considerações Finais}

A etapa prospectiva permitiu concluir que não há tecnologias com pedidos de proteção que permitam a valoração de novas tecnologias protegidas por patentes ou por registros de programa de computador, cujo processo de valoração inclua etapas de apoio aos usuários, propiciando mais adequado preenchimento das informações, além de incluir a participação, tanto do gestor de inovação, como dos inventores/autores.

Ademais, no mercado brasileiro, apesar da importância da valoração de uma tecnologia para uma negociação e transferência de tecnologia mais satisfatória para ambas as partes e com as dificuldades que existem para escolher e pôr em prática uma metodologia de valoração de forma manual, não estão disponíveis softwares com essa finalidade.

Em etapa pós-prospectiva, com elaboração de SWOT e Roadmap, é possível identificar oportunidade de mercado por meio de produto inovador, adicionadas as forças internas, com equipe contendo especialistas em desenvolvimento de sistemas, conhecimentos sobre PI e TT, além da existência de diversas fontes de dados de um dos autores do artigo para aplicação na ferramenta.

O desenvolvimento do sistema atenderá a uma necessidade interna do NIT/Univasf, mas também se trata de uma tecnologia promissora para todos os demais ambientes de inovação, tanto públicos como privados, no sentido de permitir obtenção de valores de referência para novas tecnologias, sejam produtos/processos ou programas de computador, podendo auxiliar sobremaneira os processos de negociação e a transferência de tecnologias.

\section{Perspectivas Futuras}

Estima-se que estudos com ênfase na prospecção de tecnologias licenciadas, e se estas foram previamente valoradas ou não, manual ou de forma automatizada, com uso de softwares ou planilhas eletrônicas, a partir de quantidade significativa de NITs no Brasil, podem ser relevantes para a geração de conhecimentos sobre como vêm ocorrendo os processos de negociação no Brasil. 
Acrescenta-se ainda a relevância associada ao desenvolvimento de novos métodos de valoração, que sejam cada vez mais completos quanto às especificidades inerentes às novas tecnologias, permitindo a inserção de parâmetros relativos à redução de impactos ambientais negativos, por exemplo, ou ainda, que contenham termos ligados ao nível de maturidade tecnológica ou Technology Readiness Level (TRL).

\section{Referências}

AMARAL, Hudson F. et al. Avaliação de Ativos Intangíveis: Modelos Alternativos para Determinação do Valor de Patentes. Revista de Gestão, Finanças e Contabilidade, UNEB, Salvador, v. 4, n. 1, p. 123-143, jan.-abr., 2014.

BARRO, D. N.; SANTOS, V. M. L; AZEVÊDO, L. C. Prospecção Tecnológica Acerca de Sistemas de Elaboração de Contratos de Transferência de Tecnologia. Cadernos de Prospecção, Salvador, v. 13, n. 5, p. 1.477-1.491, dez. 2020.

BRASIL. Software Público Brasileiro: Catálogo de Software Público Brasileiro. 2021. Disponível em: https://www.gov.br/governodigital/pt-br/software-publico/catalogo/catalogo. Acesso em: 28 mar. 2021.

BRITO, C. V. S. P.; SANTOS, V. M. L.; SILVA, I. R. A. Guia Prático para Valoração de patentes. Juazeiro, BA: Univasf, 2021.

BUAINAIN, Antônio M. et al. Propriedade Intelectual, Inovação e Desenvolvimento: desafios para o Brasil. Rio de Janeiro: ABPI, 2018.

CAVALCANTE, F. V.; ALMEIDA, M. B. C.; RENAULT, T. B. Intervenientes dos processos de transferência tecnológica em uma instituição de ciência e tecnologia: o caso FIOCRUZ. Revista Gestão \& Tecnologia, Pedro Leopoldo, v. 19, n. 2, p. 217-239, abr.-jun. 2019.

CERVANTES, V.; LUCARELLI, F. Inovação, Desenvolvimento e Propriedade Intelectual. Revista Juris UniToledo, Araçatuba, SP, v. 3, n. 4, p.163-176, out.-dez. 2018.

COSTA, L. S. C.; SILVA, C. T. R. A Proteção da Propriedade Intelectual de Softwares na Legislação Brasileira. In: IV SEMANA UNIVERSITÁRIA DA URCA - XXII SEMANA DE INICIAÇÃO CIENTÍFICA. 21 a 25 de outubro de 2019. Anais [...]. 2019.

CRUZ, Silvana S. et al. Transferência de Tecnologia sob a Ótica da Política de Inovação e do Marco Legal da Ciência, Tecnologia e Informação. Cadernos de Prospecção, Salvador, v. 13, n. 4, p. 1.024-1.035, 2020.

FERREIRA, A. R. F; SOUZA, A. L. R. Análise dos Procedimentos e Critérios Necessários à Valoração de Propriedade Intelectual para a Transferência de Tecnologia no Âmbito dos Núcleos de Inovação Tecnológica (NITs). Cadernos de Prospecção, Salvador, v. 12, n. 5, p. 1.012-1.039, dez. 2019.

FERREIRA, C. L. D.; GHESTI, G. C; BRAGA, P. R. S. Desafios para o Processo de Transferência de Tecnologia na Universidade de Brasília. Cadernos de Prospecção, Salvador, v. 10, n. 3, p. 341355, jul.-set. 2017.

HÜLLER, Keylha S. et al. Análise de Ponto de Função: estudo de caso para valoração de custos no desenvolvimento de um sistema computacional em NITs. Navus, Florianópolis, SC, v. 11, p. 1-18, jan.-dez. 2021. 
LATTES. Currículo Lattes: de Julio Cezar Mairesse Siluk. 2021. Disponível em: http://lattes.cnpq. br/8315298509051752. Acesso em: 28 mar. 2021.

LEITE, Rafael Â. S. et al. Valoração de Ativos de Propriedade Intelectual. In: RUSSO, S. L; SILVA, M. B.; SANTOS, V. M. L. Propriedade Intelectual e Gestão de Tecnologias. Aracaju: Associação Acadêmica de Propriedade Intelectual, 2018. p. 82-93.

NETO, José A. C. et al. Prospecção Tecnológica dos Registros de Softwares de Administração Pública. ISTI/SIMTEC, Aracaju, SE, v. 3, n.1, p. 8-17, 21 a 23 de setembro de 2016.

REIS, D. R.; VINCENZI, T. B.; PUPO, F. P. Técnicas de Prospecção: um Estudo Comparativo. RAC, Rio de Janeiro, v. 20, n. 2, art. 1, p. 135-153, mar.-abr. 2016.

SANTOS, Vivianni M. L. dos et al. Processo de Valoração de Tecnologias Protegidas por Patentes ou por Registros de Programas de Computador. Depositante: Fundação Universidade Federal do Vale do São Francisco. BR 102021007443 4. Depósito: 19/04/2021.

SARATKAR, Ajay Nanaji. An Article on Importance of Software Technologies in Business and Management Science. International Journal of Engineering Applied Sciences and Technology, [s.l.], v. 4, n. 4, p. 291-294, 2019.

SILVA, D. R.; MENDES, E. J. J.; VIANA, W. Importância da Avaliação do Produto de Software antes do Lançamento ao Mercado de Acordo com as Normas ISO/IEC 9126 e 14598. Tecnologias em Projeção, [s.l.], v. 11, n. 1, 2020.

SOUZA, Sandra S. et al. Gestão Estratégica da Propriedade Intelectual: um estudo sobre o Núcleo de Inovação Tecnológica do Instituto Federal Baiano no contexto do novo marco legal da Ciência, Tecnologia e Inovação (CT\&I). Cadernos de Prospecção, Salvador, v. 14, n. 2, p. 380-396, 2021.

UFSM - UNIVERSIDADE FEDERAL DE SANTA MARIA. Plataforma UFSM Pública: Produção do docente Julio Cezar Mairesse Siluk. 2021. Disponível em: http://ufsmpublica.ufsm.br/docente/14499/ producao. Acesso em: 28 mar. 2021.

\title{
Sobre os Autores
}

\author{
Antonio Fredson de Sá Novaes \\ E-mail: antonio.fredson@univasf.edu.br \\ ORCID: http://orcid.org/0000-0001-6531-5642 \\ Mestrando em Propriedade Intelectual de Transferência de Tecnologia para Inovação (Profnit) pela Univasf. \\ Bacharel em Ciência da Computação pela Universidade Católica de Pernambuco (UNICAP) em 1995. \\ Lattes: http://lattes.cnpq.br/4384846909675676. \\ Endereço profissional: Secretaria de Tecnologia da Informação, Universidade Federal do Vale do São Francisco, \\ Campus Sede, Av. José de Sá Maniçoba, s/n, Centro, Petrolina, PE. CEP: 56304-917.
}

\section{Gilton José Ferreira da Silva}

E-mail: gilton@dcomp.ufs.br

ORCID: http://orcid.org/0000-0002-2281-9426

Doutor em Ciência da Propriedade Intelectual pela UFS. Lattes: http://lattes.cnpq.br/9431168170232771

Endereço profissional: Departamento de Computação, Universidade Federal de Sergipe, Av. Marechal Rondon, s/n. Bairro Rosa Elze, São Cristóvão, SE. CEP: 49100-000. 


\section{Vivianni Marques Leite dos Santos}

E-mail: vivianni.santos@gmail.com

ORCID: http://orcid.org/0000-0001-8741-8888

DSc em Química. Lattes: http://lattes.cnpq.br/3040638073236492.

Endereço profissional: Profnit, Universidade Federal do Vale do São Francisco, Campus Juazeiro, Av. Antonio Carlos Magalhães, n. 510, Santo Antônio, Juazeiro, BA. CEP: 48902-300. 